\title{
Erratum to: From Fear to Anxiety: An Introduction
}

\author{
Andreja Zevnik ${ }^{1}$
}

\section{Erratum to: Law Critique \\ DOI 10.1007/s10978-017-9211-x}

The title of this article has been corrected to From Fear to Anxiety: An Introduction as it forms the introductory piece to a collection of articles making up the Special Issue From Fear to Anxiety: An exploration into a new socio-political temporality.

The online version of the original article can be found under doi:10.1007/s10978-017-9211-x.

$\triangle$ Andreja Zevnik

andreja.zevnik@manchester.ac.uk

1 Department of Politics, School of Social Sciences, University of Manchester, Oxford Road, Manchester M13 9PL, UK 